\title{
Risk indicators associated with in-hospital mortality and severity in patients with diabetes mellitus and confirmed or clinically suspected COVID-19
}

\author{
Marzieh Pazoki ${ }^{1}$ - Mohammad Keykhaei ${ }^{2} \cdot$ Samira Kafan $^{1} \cdot$ Mahnaz Montazeri $^{3}$ - Mojgan Mirabdolhagh Hazaveh ${ }^{4}$. \\ Mehran Sotoodehnia ${ }^{5}$ - Sina Kazemian ${ }^{2} \cdot$ Mohammad Talebpour $^{6} \cdot$ Haleh Ashraf $^{7,8} \cdot$ Reza Shariat Moharari $^{9}$. \\ Fazeleh Majidi ${ }^{8} \cdot$ Nazanin Hedayati Amlashi $^{8} \cdot$ Sara Zare $^{8} \cdot$ Maryam Fathi $^{8} \cdot$ Zohre Azimbeik $^{8} \cdot$ Mehdi Ebrahimi $^{4}$
}

Received: 27 September 2020 / Accepted: 22 November 2020 / Published online: 7 January 2021

(C) Springer Nature Switzerland AG 2021

\begin{abstract}
Purpose This study aims to investigate risk indicators of in-hospital mortality and severity of coronavirus disease-2019 (COVID19) in patients with diabetes mellitus (DM).

Methods In this retrospective study, we studied patients with COVID-19 referred to Sina Hospital, Tehran, Iran, from February 20 to May 14, 2020. Patients with either a positive real-time reverse-transcriptase polymerase-chain-reaction test of swab specimens or high clinical suspicion according to the World Health Organization interim guidance were included. We accurately divided all patients into two groups based on diabetes affection and followed-up patients with DM based on incurring death, severe COVID-19, and in-hospital complications.

Results We enrolled 574 patients with COVID-19 in the final analysis, of whom 176 (30.7\%) patients had DM. In this study, 104 $(18.1 \%)$ patients deceased, and 380 (66.2\%) patients incurred severe COVID-19. We found that COVID-19 patients with DM had a significantly higher mortality rate $(P$ value $<0.001)$, severe disease $(P$ value $<0.001)$, and in-hospital complications (all $P$ values $<0.05$ ). Besides that, in patients with $\mathrm{DM}$, admission temperature (odds ratio $(\mathrm{OR}): 1.69$, $\mathrm{P}$ value: 0.024$)$, oxygen saturation (OR: 0.92, P value: 0.004), and urea (OR: 1.01, P value: 0.048 ) were independent risk indicators of in-hospital mortality. In addition, subgroup analysis of diabetic patients based on admission glucose level showed significant differences between these groups regarding acute cardiac injury ( $P$ value: 0.044$)$ and acute liver injury (P value: 0.002$)$.

Conclusions Patients with DM admitted with lower oxygen saturation, elevated temperature, and higher urea are more susceptible to progress to more severe COVID-19 and poor prognosis. This indicates a necessity for more precise care during hospitalization for these patients.
\end{abstract}

Keywords COVID-19 $\cdot$ Diabetes mellitus $\cdot$ Hyperglycemia $\cdot$ Mortality $\cdot$ Severity

Mehdi Ebrahimi

M_ebrahimi49@yahoo.com

1 Department of Pulmonary Medicine, Sina Hospital, Tehran University of Medical Sciences, Tehran, Iran

2 Students' Scientific Research Center (SSRC), Tehran University of Medical Sciences, Tehran, Iran

3 Department of Infectious Diseases, Sina Hospital, Tehran University of Medical Sciences, Tehran, Iran

4 Department of Endocrinology Medicine, Sina Hospital, Tehran University of Medical Sciences, Hasan Abad Sq, Tehran, Iran
5 Department of Emergency Medicine, Sina Hospital, Tehran University of Medical Sciences, Tehran, Iran

6 Department of Surgery, Sina Hospital, Tehran University of Medical Sciences, Tehran, Iran

7 Cardiac Primary Prevention Research Center (CPPRC), Cardiovascular Diseases Research Institute, Tehran University of Medical Sciences, Tehran, Iran

8 Research Development Center, Sina Hospital, Tehran University of Medical Sciences, Tehran, Iran

9 Department of Anesthesiology and Critical Care, Sina Hospital, Tehran University of Medical Sciences, Tehran, Iran 


\section{Introduction}

Since December 2019, the novel coronavirus disease 2019 (COVID-19), caused by severe acute respiratory syndrome coronavirus 2 (SARS-COV-2), has received much attention due to its global effect. The disease evolved rapidly worldwide and was declared a pandemic by the World Health Organization (WHO) on March 11, 2020 [1]. By November 8,2020 , the virus has caused 50,395,239 confirmed cases and $1,258,235$ deaths worldwide [2]. Also, with 682,486 confirmed cases and 38,291 deaths, Iran is considered as one of the most affected countries during the COVID-19 pandemic [2]. The clinical manifestations of COVID-19 are various, including acute respiratory distress syndrome (ARDS), pulmonary edema, pneumonia, acute kidney injury (AKI), and multiple organ failure; however, the majority of patients present with mild or without any symptoms [3]. According to recent systematic reviews, diabetes mellitus (DM), hypertension (HTN), and cardiovascular diseases were the most prevalent comorbidities observed in patients with COVID-19 [4, 5]. Besides, several studies have highlighted that patients with underlying diseases, encompassing DM and HTN, are more prone to develop severe diseases during COVID-19, which shed some light on the essential role of these comorbidities in COVID-19 progression [4, 6, 7].

DM is one of the most common metabolic diseases that cause short-term and long-term complications. In 2019, DM contributed to more than 1.5 million deaths and 70.8 million disability-adjusted life-years globally [8]. It is caused by the immune system destroying pancreatic cells or malformations that result in resistance to insulin action [9]. In patients with DM, excess adipose tissue can cause chronic inflammatory and pro-oxidative states; this inflammation alongside with hyperglycemia can lead to relative immune-suppression and so the patients may be prone to respiratory tract viral infections, including H1N1 influenza and COVID-19 [10, 11]. In support of this concept, a recent meta-analysis represented that patients with COVID-19 and DM had higher mortality compared with patients without DM [6]. Moreover, According to a study conducted by Singh AK, et al., there is an association between poorly-controlled hyperglycemia and increased both mortality and severity in patients with SARS-CoV-2 disease [7]. So far, the plausible risk factors of COVID-19 development in patients with DM have not been definitely explained, whereas several studies have reported the relation between DM and COVID-19 [6, 12, 13]. In addition, despite the major concerns about the association between admission glucose level with progression and in-hospital outcomes in patients with DM and COVID-19, comprehensive published data on this issue are lacking.

In this article, considering the importance of the topic, first, we aim to retrospectively study the association between diabetes mellitus and poor outcomes in patients with COVID-19.
Moreover, the essential purpose of our study is to investigate risk indicators of in-hospital mortality and severity of COVID-19 in patients with DM. Last but not least, we compared in-hospital outcomes of COVID-19 in patients with DM based on the admission glucose level.

\section{Methods and materials}

\section{Ethical considerations}

The protocol of this study was approved by the Ethics Committee of Tehran University of Medical Sciences (IR.TUMS.VCR.REC.1399.005). All participants or their legal guardians gave written informed consent before inclusion in the study.

\section{Study design and participants}

In this study, patients with confirmed or clinically suspected COVID-19 who were admitted to Sina Hospital from February 20 to May 14, 2020, enrolled. Sina Hospital is one of the major tertiary teaching hospitals affiliated by Tehran University of Medical Sciences, and it is a designated hospital for treating COVID-19 in the capital Tehran.

We included 611 patients $\geq 18$ years of age with confirmed or clinically suspected COVID-19 who met one of the following criteria: 1) Positive real-time reverse-transcriptase polymerase-chain-reaction (PCR) test of oropharyngeal or endotracheal swab specimens. 2) Highly suspicious patients according to the WHO interim guidance and Iranian national committee of COVID-19, including patients with groundglass opacity alone or ground-glass opacity accompanied with consolidation in chest computed tomography, not completely explained by lobar collapse, volume overload or nodules in company with the history compatible with COVID-19 [14, 15]. The algorithm of patient care for adults presenting with respiratory symptoms to Sina Hospital emergency department has been published before [16]. We excluded 37 patients due to in-hospital transmission or lack of key information in their medical records, and 574 patients entered the final analysis. With regard to the impacts of DM on the prognosis of patients with COVID-19, first, we accurately divided all patients into two groups: patients with $\mathrm{DM}(N=176)$ and patients without DM $(N=398)$. Afterward, patients with DM entered the final analysis based on incurring death, severe COVID-19, and inhospital complications.

The demographics and clinical data of patients included in this study were obtained from patients' electronic medical records. We evaluated patients based on demographics, their past medical history, drug history, admission vital signs, admission laboratory data, and in-hospital outcomes. Routine blood tests were measured in the laboratory of Sina hospital. 


\section{Definitions}

Diabetes mellitus was defined as one of the following: (1), fasting blood sugar (FBS) $\geq 126 \mathrm{mg} / \mathrm{dL}(7.0 \mathrm{mmol} / \mathrm{L})$ on two occasions, or(2), two-hour plasma glucose $\geq 200 \mathrm{mg} / \mathrm{dL}$ $(11.1 \mathrm{mmol} / \mathrm{L})$ during the oral glucose tolerance test on two occasions, or( 3 ), hemoglobin A $1 \mathrm{c}(\mathrm{HbA} 1 \mathrm{c}) \geq 6.5 \%$ ( $47.5 \mathrm{mmol} / \mathrm{mol}$ ), or (4), a random plasma glucose $\geq 200 \mathrm{mg} /$ $\mathrm{dL}(11.1 \mathrm{mmol} / \mathrm{L})$ in a patient with classic symptoms of hyperglycemia or hyperglycemic crisis, or(5), positive history of anti-diabetic medication use, according to the latest American Diabetic Association guidelines [17]. Poor controlled DM was defined as $\mathrm{FBS} \geq 126 \mathrm{mg} / \mathrm{dL}(7.0 \mathrm{mmol} / \mathrm{L}), \mathrm{HbA} 1 \mathrm{c}>7 \%$ (53 $\mathrm{mmol} / \mathrm{mol}$ ), or blood sugar $(\mathrm{BS}) \geq 200 \mathrm{mg} / \mathrm{dL}$ (11.1 mmol/L). Impaired fasting glucose (IFG) was described as $\mathrm{FBS} \geq 100$ and $<126 \mathrm{mg} / \mathrm{dL}$ ( $\geq 5.6$ and $<7 \mathrm{mmol} / \mathrm{L}$ ), and Impaired glucose tolerance(IGT) was defined by an elevated 2-h plasma glucose concentration $\geq 140$ and $<200 \mathrm{mg} / \mathrm{dL}$ $(\geq 7.8$ and $<11.1 \mathrm{mmol} / \mathrm{L}$ ) after a 75 -g glucose load. Hypertension was clarified as the systolic blood pressure (SBP) $\geq 140 \mathrm{mmHg}$ or diastolic blood pressure (DBP) $\geq 90 \mathrm{mmHg}$ or history of anti-hypertensive treatment [18]. Body mass index (BMI) was measured as weight, divided by height squared $\left(\mathrm{kg} / \mathrm{m}^{2}\right)$.

Cardiac disease was elucidated as a history of heart failure, or coronary artery disease (stenosis of coronary artery $\geq 50 \%$ ), or taking treatment for any of these conditions. History of stroke or transient ischemic attack (TIA) was determined as a cerebrovascular disease. Patients with a history of interstitial lung disease, asthma, or chronic obstructive pulmonary disease were classified as chronic lung disease. Chronic kidney disease (CKD) was defined as renal replacement requirement or a glomerular filtration rate below $30 \mathrm{ml} / \mathrm{h}$. Patients with a history of treated neoplasm considered a positive malignancy history. ARDS was described according to the Berlin definition criteria [19]. Acute cardiac injury (ACI) was diagnosed if serum level of high sensitive cardiac troponin I (hs-cTnI) was above the 99th percentile upper reference limit $(11 \mathrm{pg} / \mathrm{mL}$ for women and $26 \mathrm{pg} / \mathrm{mL}$ for men) [20]. AKI was determined as an increase in serum creatinine $(\mathrm{Cr})$ to $\geq 1.5$ times baseline within the prior 7 days, or Urine volume $<0.5 \mathrm{ml} / \mathrm{kg} / \mathrm{h}$ for $6 \mathrm{~h}$, or an increase in serum creatinine by $\geq 0.3 \mathrm{mg} / \mathrm{dl}$ ( $>26.5 \mu \mathrm{mol} / \mathrm{l}$ ) within $48 \mathrm{~h}$ except for patients with endstage renal disease [21]. Acute liver injury (ALI) was defined as an increase in serum levels of alanine aminotransferase (ALT) or aspartate aminotransferase (AST) greater than 3 units above upper limit normal (ULN), alkaline phosphatase (ALP), or total bilirubin $\geq 2 x U L N$ [22]. The neutrophil to lymphocyte ratio (NLR) was calculated by dividing the absolute neutrophil count by the lymphocyte count, and platelet-to-lymphocyte ratio (PLR) was computed by dividing the absolute platelet count by the lymphocyte count. The systemic immuneinflammation index (SII) was measured by (neutrophil count x platelets)/ (lymphocyte count). Patients with one of the following features, considered to have a severe disease: oxygen saturation $\leq 93$, or $>50 \%$ lung involvement on imaging, dyspnea, septic shock, respiratory failure, or multiple organ dysfunction/failure. The remaining patients were categorized as non-severe COVID-19. We defined these criteria similar to Wu and colleagues' study and modified it to compare patients in severe versus non-severe COVID-19 [23]. Multiple organ dysfunction was determined as patients with at least two complications, including ARDS, ACI, AKI, ALI. Positive drug history was considered as taking medication for at least one month before admission.

\section{Statistical analysis}

Categorical variables were presented as numbers and percentages (\%) and analyzed using Fisher's exact test and chisquared test. Continuous variables with normal distribution were analyzed using independent samples T-test and were expressed as mean \pm standard deviation (SD). Variables without normal distribution which were identified by using the Kolmogorov-Smirnov test result presented as median (interquartile range). Continuous variables with skewed distribution were analyzed using the Mann-Whitney U test. In this study, multivariate binary logistic regression was carried out to explore the independent risk indicators associated with inhospital mortality and to eliminate the effect of confounders. In addition, we performed a regression analysis only in patients with a positive PCR test. All statistical analyses were performed using the SPSS 21.0 software. In our study, $P$ value $<0.05$ was considered statistically significant.

\section{Results}

\section{Demographics, clinical and laboratory data}

In the present study, we included 574 patients with COVID19 , including $176(30.7 \%)$ patients with DM in the final analyses. Of all patients, 306 (53.3\%) underwent swab PCR test, of whom $135(23.5 \%)$ patients were definitely diagnosed with COVID-19. The swab PCR test was done for 114 (64.7\%) patients with DM, of which 57 (32.4\%) specimens were positive for COVID-19. Overall, 135 (23.5\%) patients in the whole study and 57 (32.4\%) patients with DM were definitely diagnosed with COVID-19 based on a positive swab PCR test. It should be noted that despite this point that it was not feasible to perform the PCR test for other patients included in our study, they were highly suspicious for COVID-19 based on the national and international guidelines $[14,15]$.

The mean age was 56.8 years (interquartile range: 44.0 69.0 years), and 217 (37.8\%) were female. The most common comorbidities were HTN (39.4\%) and DM (30.6\%). ARDS 
was the most frequent complication revealed in $28.7 \%$ of all patients. During hospitalization, $104(18.1 \%)$ patients deceased, and $380(66.2 \%)$ patients developed severe disease. Although the data of history, mortality, severity, ARDS, and invasive ventilation were complete and the rate of missing data for most of the laboratory data is less than $5 \%$, we had not the data of lactate dehydrogenase (LDH), erythrocyte sedimentation rate (ESR), hs-cTnI, and liver transaminases in $27.8 \%, 11.8 \%, 26.7 \%$, and $20.8 \%$ of patients.

The baseline characteristics of the study population are shown in (Table 1). Patients with DM were significantly older, had higher BMI, and presented with lower $\mathrm{O}_{2}$ saturation on admission compared to patients without DM. History of comorbidities was more prevalent in patients with DM.
Furthermore, they were at increased risk of mortality and developed more severe COVID-19. In addition, in-hospital complications occurred considerably higher in patients with DM than patients without diabetes.

The associated risk indicators of in-hospital severity and mortality for COVID-19 in patients with DM are illustrated in (Table 2). During follow-up, 54 (30.7\%) patients with DM deceased, and 135 (76.7\%) developed severe disease. Our study showed that the patients in the deceased group were significantly older than the others. According to patients' drug history, there is no association between the use of any medication and COVID-19 progression. In terms of vital signs, both mortality and severity were significantly higher in
Table 1 Admission

characteristics and clinical outcomes of patients with and without DM

\begin{tabular}{|c|c|c|c|c|}
\hline Characteristic $^{\dagger}$ & $\begin{array}{l}\text { Total } \\
(n=574)\end{array}$ & $\begin{array}{l}\text { Diabetes } \\
(n=176)\end{array}$ & $\begin{array}{l}\text { Non-diabetes } \\
(n=398)\end{array}$ & $\mathbf{P}^{*}$ \\
\hline \multicolumn{5}{|l|}{ Demographics } \\
\hline Age & $56.8 \pm 16.3$ & $65.0 \pm 12.1$ & $53.2 \pm 16.7$ & $<0.001$ \\
\hline $\begin{array}{l}\text { Female } \\
\text { Male }\end{array}$ & $\begin{array}{l}217.0(37.8 \%) \\
357.0(62.2 \%)\end{array}$ & $\begin{array}{l}77.0(43.8 \%) \\
99.0(56.3 \%)\end{array}$ & $\begin{array}{l}140.0(35.2 \%) \\
258.0(64.8 \%)\end{array}$ & 0.051 \\
\hline BMI $\left(\mathrm{kg} / \mathrm{m}^{2}\right)$ & $27.4 \pm 4.7$ & $28.3 \pm 4.9$ & $26.9 \pm 4.5$ & 0.010 \\
\hline \multicolumn{5}{|l|}{ Comorbidities } \\
\hline Hypertension & $226.0(39.4 \%)$ & $111.0(63.1 \%)$ & $115.0(28.9 \%)$ & $<0.001$ \\
\hline Cardiac disease & $118.0(20.6 \%)$ & $56.0(31.8 \%)$ & $62.0(15.6 \%)$ & $<0.001$ \\
\hline Cerebrovascular disease & $18.0(3.1 \%)$ & $15.0(8.5 \%)$ & $3.0(0.8 \%)$ & $<0.001$ \\
\hline Chronic lung disease & $39.0(6.8 \%)$ & $12.0(6.8 \%)$ & $27.0(6.8 \%)$ & 0.988 \\
\hline Chronic kidney disease & $30.0(5.2 \%)$ & $19.0(10.8 \%)$ & $11.0(2.8 \%)$ & $<0.001$ \\
\hline Malignancy & $20.0(3.5 \%)$ & $9.0(5.1 \%)$ & $11.0(2.8 \%)$ & 0.157 \\
\hline \multicolumn{5}{|l|}{ Vital signs } \\
\hline Heart rate & $88.3 \pm 16.7$ & $89.0 \pm 14.3$ & $88.0 \pm 17.7$ & 0.530 \\
\hline Respiratory rate & $20.5 \pm 8.7$ & $20.3 \pm 6.0$ & $20.6 \pm 9.7$ & 0.793 \\
\hline SBP & $123.8 \pm 20.4$ & $125.9 \pm 21.7$ & $122.8 \pm 19.8$ & 0.134 \\
\hline DBP & $75.9 \pm 11.5$ & $75.9 \pm 11.3$ & $75.9 \pm 11.6$ & 0.987 \\
\hline Temperature $\left({ }^{\circ} \mathrm{C}\right)$ & $37.2 \pm 1.5$ & $37.2 \pm 0.9$ & $37.1 \pm 1.7$ & 0.509 \\
\hline Oxygen saturation (\%) & $90.7 \pm 7.5$ & $89.5 \pm 7.7$ & $91.2 \pm 7.4$ & 0.016 \\
\hline \multicolumn{5}{|l|}{ In-hospital outcomes } \\
\hline Hospital length of stay (day) & $4.0(2.0-7.0)$ & $5.0(3.0-8.0)$ & $3.0(1.0-6.0)$ & $<0.001$ \\
\hline Severity & $380.0(66.2 \%)$ & $135.0(76.7 \%)$ & $245.0(61.6 \%)$ & $<0.001$ \\
\hline Mortality & $104.0(18.1 \%)$ & $54.0(30.7 \%)$ & $50.0(12.6 \%)$ & $<0.001$ \\
\hline ARDS & $165.0(28.7 \%)$ & $68.0(38.6 \%)$ & $97.0(24.4 \%)$ & $<0.001$ \\
\hline Invasive ventilation & $69.0(12.0 \%)$ & $34.0(19.3 \%)$ & $35.0(8.8 \%)$ & $<0.001$ \\
\hline $\mathrm{ACI}$ & $114.0(19.9 \%)$ & $45.0(25.6 \%)$ & $69.0(17.3 \%)$ & 0.023 \\
\hline AKI & $69.0(12.0 \%)$ & $37.0(21.0 \%)$ & $32.0(8.0 \%)$ & $<0.001$ \\
\hline ALI & $55.0(9.6 \%)$ & $21.0(11.9 \%)$ & $34.0(8.5 \%)$ & 0.203 \\
\hline Multiorgan damage & $105.0(18.3 \%)$ & $46.0(26.1 \%)$ & $59.0(14.8 \%)$ & 0.001 \\
\hline
\end{tabular}

$\dagger$ Data are presented as mean \pm standard deviation, number (\%), or median (interquartile range)

* Statistically significant $P$-values are bolded

ACI: acute cardiac injury; AKI: acute kidney injury; ALI: acute liver injury; ARDS: acute respiratory distress syndrome; BMI: body mass index; DBP: diastolic blood pressure; SBP: systolic blood pressure 


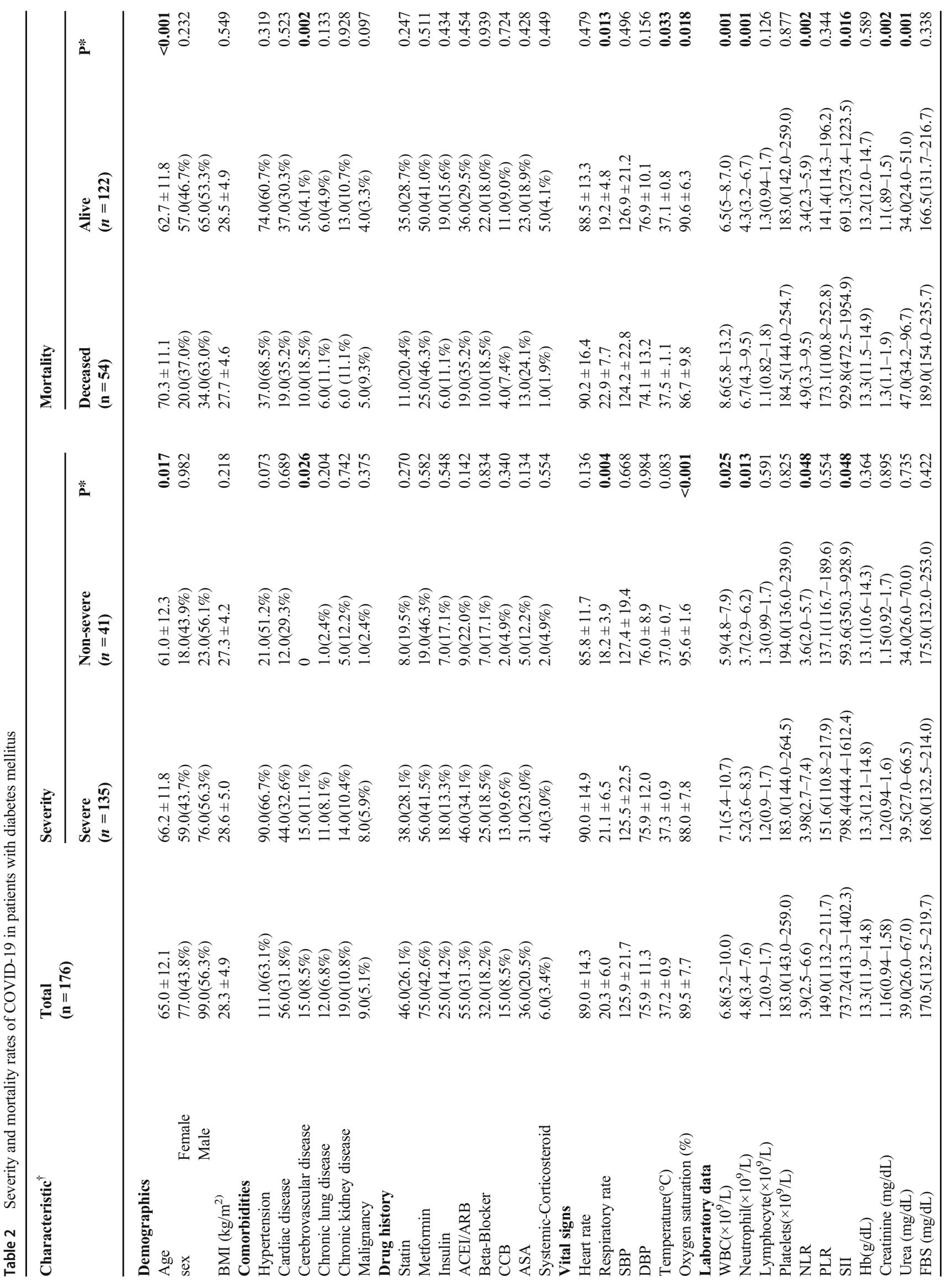




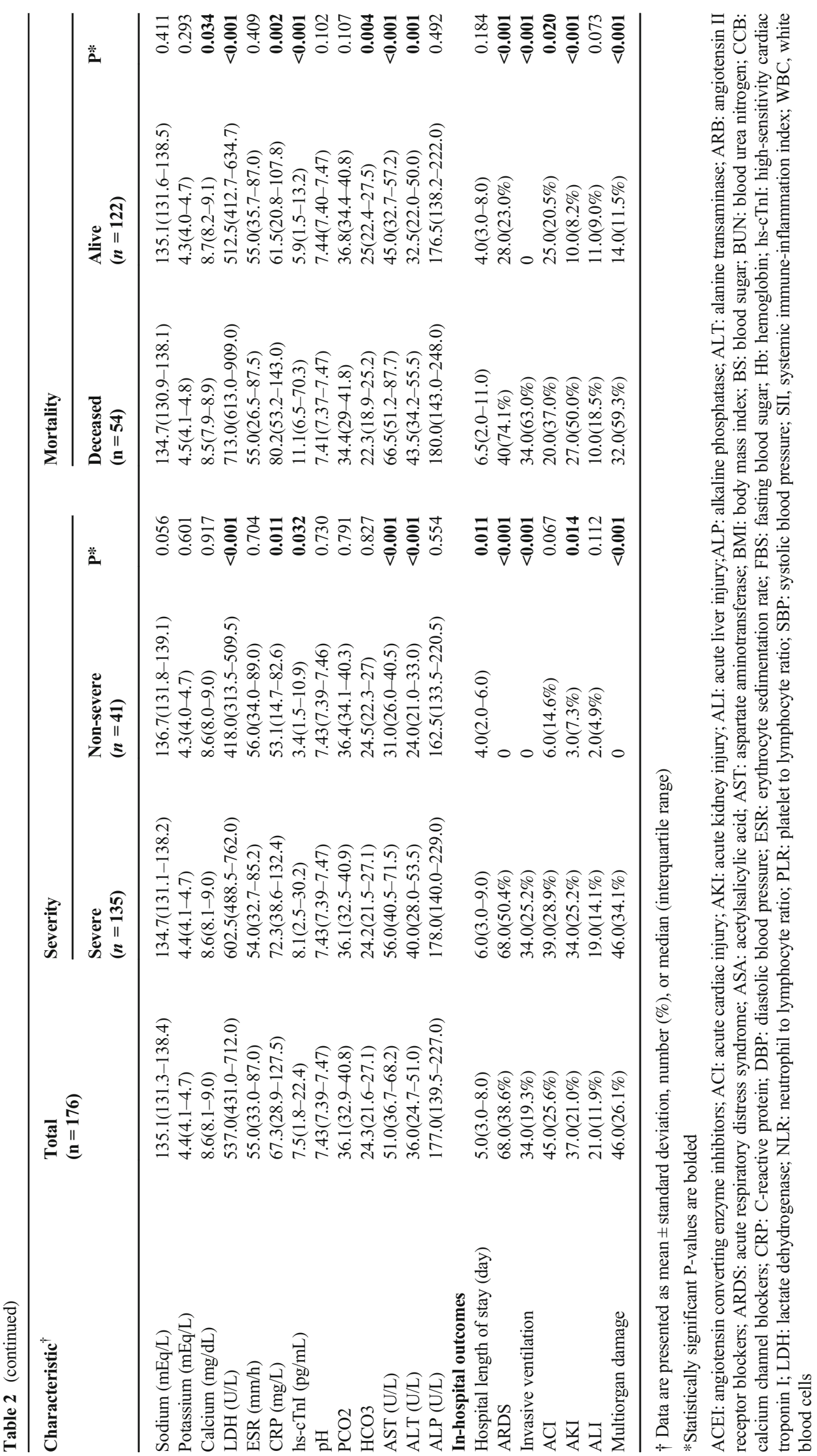


patients with DM who had a higher respiratory rate and lower oxygen saturation on admission. The body temperature on admission was only considerably higher in the deceased group ( $P$ value: 0.033$)$. In our study, laboratory tests show that higher white blood cell (WBC), inflammatory biomarkers, and liver function tests were associated with an increased risk of both mortality and severity in patients with DM. All complications except for ALI were associated with both higher in-hospital mortality and more severe disease.

\section{Association between DM control status and clinical outcomes}

We divided patients with DM into three sub-groups based on their glucose level on admission, which included 27 patients with controlled glucose, 30 patients with impaired glucose, and 112 patients with poorcontrolled glucose (Table 3). In this analysis, seven patients with DM were excluded due to a lack of information about their admission blood sugar status. There were statistically significant differences between these groups regarding ACI and ALI, while all patients with ALI were in the poor-controlled group ( $P$ value: 0.002$)$. Patients with impaired and poor-controlled glucose were more likely to develop ACI comparing to patients with controlled glucose ( $\mathrm{P}$ value: 0.044 ), but there was no significant difference between these two groups. Also, there is an increasing trend that severe manifestation is more prevalent in patients with a higher glucose level on admission comparing to controlled glucose patients ( $P$ value: 0.052). Patients were similar in terms of age, gender, and history of comorbidities between these groups (supplementary Table 1).

\section{Multivariant regression models for in-hospital mortality}

In order to evaluate independent risk indicators of in-hospital mortality in patients with DM and COVID-19, we included 176 patients in the multivariant binary logistic regression adjusted for age, gender, history of cerebrovascular disease, temperature, oxygen saturation, NLR, urea, CRP (Table 4). In this study, admission temperature (odds ratio (OR): $1.69,95 \%$ confidence interval (CI): $1.07-2.66$; $P$ value: 0.024$)$, admission oxygen saturation (OR: $0.92,95 \%$ CI: $0.87-0.97$; $\mathrm{P}$ value: 0.004 ) and serum urea (OR: $1.01,95 \%$ CI: $1.00-1.03$; P value: 0.048 ) were independent risk indicators of in-hospital mortality. We also repeated the same analysis only in patients with DM and positive PCR tests, and only admission oxygen saturation (OR: 0.88, 95\% CI: 0.79-0.98; P value: 0.018 ) was independently associated with higher in-hospital mortality.

\section{Discussion}

In this study, we evaluated 574 patients with confirmed or clinically suspected COVID-19, including 176 (30.7\%) patients with DM. After adjusting for possible confounders, higher admission temperature (OR: 1.69), lower admission oxygen saturation (OR: 0.92), and higher urea (OR: 1.01) were independent risk indicators of in-hospital mortality of COVID-19 in patients with DM. In-hospital complications, including ACI and ALI, were notably higher in patients with poor-controlled and impaired glucose status comparing to patients with DM and normal glucose levels. Besides, patients with DM had remarkably more comorbidities and mortality rate $(30.7 \%$ vs. $12.6 \% ; P<0.001)$ and developed a more
Table 3 Comparison of inhospital outcomes of COVID-19 in patients with DM based on the admission glucose level

\begin{tabular}{|c|c|c|c|c|c|}
\hline $\begin{array}{l}\text { In-hospital } \\
\text { outcome }^{\dagger}\end{array}$ & $\begin{array}{l}\text { Total } \\
(N=169)\end{array}$ & $\begin{array}{l}\text { Controlled } \\
\text { glucose } \\
(N=27)\end{array}$ & $\begin{array}{l}\text { Impaired } \\
\text { glucose } \\
(N=\mathbf{3 0})\end{array}$ & $\begin{array}{l}\text { Poor controlled } \\
\text { glucose } \\
(N=112)\end{array}$ & $\mathbf{P}^{*}$ \\
\hline Mortality & $51(30.2 \%)$ & $12(44.4 \%)$ & $7(23.3 \%)$ & $32(28.6 \%)$ & 0.182 \\
\hline Severity & $130(76.9 \%)$ & $16(59.3 \%)$ & $23(76.7 \%)$ & $91(81.3 \%)$ & 0.052 \\
\hline ARDS & $66(39.1 \%)$ & $8(29.6 \%)$ & $13(43.3 \%)$ & $45(40.2 \%)$ & 0.523 \\
\hline Invasive ventilation & $34(20.1 \%)$ & $6(22.2 \%)$ & $5(16.7 \%)$ & $23(20.5 \%)$ & 0.857 \\
\hline ACI & $45(26.6 \%)$ & $2(7.4 \%)$ & $10(33.3 \%)$ & $33(29.5 \%)$ & 0.044 \\
\hline AKI & $36(21.3 \%)$ & $8(29.6 \%)$ & $8(26.7 \%)$ & $20(17.9 \%)$ & 0.297 \\
\hline ALI & $21(12.4 \%)$ & 0 & 0 & $21(18.8 \%)$ & 0.002 \\
\hline Multiorgan damage & $46(27.2 \%)$ & $4(14.8 \%)$ & $8(26.7 \%)$ & $34(30.4 \%)$ & 0.265 \\
\hline
\end{tabular}

$\uparrow$ Data are presented as number (\%)

*Statistically significant P-values are bolded

ACI: acute cardiac injury; AKI: acute kidney injury; ALI: acute liver injury; ARDS: acute respiratory distress syndrome 
Table 4 Logistic regression analysis for indicators of inhospital mortality of COVID-19 patients with DM

\begin{tabular}{|c|c|c|c|c|c|c|}
\hline & \multicolumn{3}{|l|}{ Model $1^{\dagger}$} & \multicolumn{3}{|l|}{ Model $2^{\ddagger}$} \\
\hline & Odds ratio & $95 \% \mathrm{CI}$ & $\mathbf{P}^{*}$ & Odds ratio & $95 \% \mathrm{CI}$ & $\mathbf{P}^{*}$ \\
\hline Age & 1.06 & $1.03-1.09$ & $<0.001$ & 1.03 & $1.00-1.07$ & 0.081 \\
\hline Sex & 1.49 & $0.77-2.87$ & 0.234 & & & \\
\hline Cerebrovascular disease & 5.32 & $1.72-16.43$ & 0.004 & & & \\
\hline Temperature $\left({ }^{\circ} \mathrm{C}\right)$ & 1.52 & $1.02-2.25$ & 0.038 & 1.69 & $1.07-2.66$ & 0.024 \\
\hline Oxygen saturation (\%) & 0.94 & $0.89-0.98$ & 0.007 & 0.92 & $0.87-0.97$ & 0.004 \\
\hline NLR & 1.12 & $1.03-1.22$ & 0.008 & & & \\
\hline Urea (mg/dL) & 1.02 & $1.01-1.03$ & $<0.001$ & 1.01 & $1.00-1.03$ & 0.048 \\
\hline CRP (mg/L) & 1.01 & $1.00-1.02$ & 0.003 & & & \\
\hline
\end{tabular}

* Statistically significant P-values are bolded

$\dagger$ Univariate binary logistic regression

$\ddagger$ Multivariate binary logistic regression adjusted for age, gender, cerebrovascular disease, temperature, oxygen saturation, NLR, urea, CRP

CRP: C-reactive protein; NLR: neutrophil to lymphocyte ratio severe form of the disease $(76.7 \%$ c vs. $61.6 \%$; $\mathrm{P}<0.001)$ comparing to patients without DM.

SARS-CoV-2 enters human cells when its spike protein binds to the angiotensin-converting enzyme 2 (ACE2) receptor [24]. ACE2 receptor is expressed in the kidney epithelium, pancreas, enterocytes, endothelial cells, lungs, and heart, and it breaks down angiotensin II to angiotensin (1-7) and also has an anti-inflammatory effect by breaking down the Angiotensin II $[25,26]$. The great expression of the ACE2 receptor after immunostaining in the exocrine and endocrine tissue of the pancreas indicates that this organ is a potential target for SARS-CoV-2 as well [27]. Therefore, pancreatic damage in patients with COVID-19 caused by virus-induced cytopathic effect can dysregulate glucose metabolism and may lead to hyperglycemia and poor prognosis in these patients.

DM is a chronic inflammatory disease caused by the impairment of insulin secretion or defects in insulin action. Accumulation of advanced glycation end products (AGEs) and activation of pro-inflammatory mediators reduce the activity of neutrophils and macrophages, resulting in facilitated pathogen replication [28]. Overall, DM is associated with reduced viral clearance, immune dysfunction, and increased susceptibility to inflammation [29]. Consistent with this concept, in a meta-analysis of 83 studies, Mantovani A, et al. [13] reported that patients with DM and COVID-19 had a significantly higher mortality rate and developed to a more critical form of the disease compared with patients without DM. In another study, patients with COVID-19 and DM had an increased risk of mortality and intensive care unit admission [30]. Similarly, Wu ZH and colleagues [6] found that DM increases the mortality rate of COVID-19 with a pooled OR of 1.75 ( $P$ value: 0.0002$)$. It is noteworthy to note that our results corroborate these studies' findings, which showed an increased risk of mortality and severity among patients with DM compared with patients without DM. Furthermore, we found that diabetic patients had significantly higher comorbidities than others, which is in line with that of Shi Q, et al. [30] who found a greater prevalence of hypertension, cardiovascular diseases, and cerebrovascular diseases among COVID-19 patients with DM.

Since the beginning of the pandemic, many studies have represented vital signs as key indicators of patients' symptoms assessment [4, 31]. Among vital signs, oxygen saturation and body temperature have the most important relationship with the severity of the disease and have been widely considered in decision making by COVID-19 patients [32]. In a recent study conducted by Liu W, et al. [33], maximum body temperature at admission was associated with COVID-19 progression (OR: 8.99). Also, in a recent study on 140 patients with COVID-19, lower oxygen saturation after oxygen supplementation was associated with a higher mortality rate [34]. Similarly, According to our results, after adjusting with possible confounders (Table 4), patients with DM who deceased $(N=54)$ during hospitalization had significantly lower oxygen saturation $(87.5 \%$ vs. $90.6 \% ; P=0.018)$ and higher body temperature $\left(37.5^{\circ} \mathrm{C}\right.$ vs. $\left.37.1^{\circ} \mathrm{C} ; P=0.033\right)$ on admission. Taken together, these findings provide useful evidence that patients with lower oxygen saturation and higher temperature at admission need to be observed more carefully in order to avoid poor outcomes.

In the kidney, proximal straight tubule cells are host cells targeted for SARS-CoV-2 and may cause an AKI [35]. Moreover, the ACE-2- SARS-CoV-2 binding leads to an increase in the ACE/ACE2 ratio, which is accompanied by oxidative stress and renal dysfunction [36]. According to a study 
conducted by Cheng Y, et al., indicators of kidney injury, including serum creatinine and blood urea nitrogen (BUN), were associated with a higher risk of mortality in patients with COVID-19 [37]. Recently, there has been evidence of an increase in mortality rate in COVID-19 patients with increased serum BUN levels $[38,39]$. A meta-analysis on 40 studies revealed that there is a positive association between higher levels of BUN and mortality rate in patients with COVID-19 [39]. Also, our results demonstrated that patients with DM who have higher serum urea $(47.0$ vs. $34.0 \mathrm{mg} / \mathrm{dL} ; P=$ $0.001)$ are at higher risk of in-hospital mortality from COVID-19. This association remained significant after adjusting with possible confounders (Table 4).

Most notably, our study investigates the association between admission glucose level with progression and inhospital outcomes in patients with DM and COVID-19. We know from previous studies that DM in COVID-19 patients can contribute to rapid progression and poor prognosis of COVID-19 [7, 24, 36, 37]. In a recent study, Raoufi and colleagues [40] studied 117 patients with coexistent DM and COVID-19. Based on the HbAlc value, patients were divided into two groups: well-controlled and poorly-controlled DM status. They found that severity outcomes and radiologic severity scores were similar between these groups. On the other hand, in another study by Iacobellis and colleagues [41], hyperglycemia on the first day of admission regardless of DM history was the best predictor of radiographic imaging of SARS-CoV-2. Zhang and colleagues [42] studied 166 patients with COVID-19; they concluded that hyperglycemia in both $\mathrm{DM}$ and secondary hyperglycemia patients with COVID was associated with increased inflammation-related biomarkers and poor prognosis.

In our study, we compared the impact of admission glucose level on disease progression. Patients with uncontrolled blood sugar were more likely to have a severe disease $(P=0.051)$. Also, in-hospital complications, including ACI and ALI, were significantly higher in patients with poor-controlled and impaired glucose status comparing to patients with DM who were admitted with normal blood glucose. While mean age, gender, and history of underlying diseases were similar between these groups (supplementary Table 1). The higher incidence of ACI and ALI in patients with DM who presented with hyperglycemia may be explained by the reason that DM and hyperglycemia can lead to elevated expression of ACE2 receptor [43]; at the same time, some studies have found the ACE2 receptor in different organs such as the heart, pancreas, lungs, and cholangiocytes [44]. Strikingly, our findings are nearly consistent with the study conducted by Zhu, et al., which indicated that COVID-19 patients who have poorlycontrolled diabetes are more prone to develop complications including AKI and acute heart injury compared with patients with well-controlled diabetes even after adjustment for possible confounders [45]. Therefore, patients with early hyperglycemia can be prone to develop multiorgan injury during COVID-19 progression.

All in all, whether patients with DM are more susceptible to COVID-19 than other patients are still unclear. But many studies demonstrated that DM contributes to adverse prognosis and more severe disease in patients with COVID-19 [36, $42,46,47]$. So far, two studies [41, 42] have indicated that patients with early hyperglycemia are prone to rapid and more severe progression of COVID-19. In addition to our findings, treatment for hyperglycemia in patients with DM, especially those with lower oxygen saturation and higher body temperature, might be crucial, and more supportive care must be considered in these patients.

\section{Limitations}

We would like to emphasize that our study has several limitations; however, we believe our study population is representative of patients diagnosed in Sina hospital. First, it is a retrospective study with possible biases that requires caution to interpret its results. Second, it is a single-center observational study on the Iranian population, which has its inherent biases. Third, the sample size of some biomarkers, including LDH, ESR, hs-cTnI, and liver transaminases, was small, which may result in under-or overestimation of ALI and ACI. Forth, multivariable cox regression analysis seems to be the best approach for evaluating associations between the characteristics and outcomes of the patients; although, we performed binary logistic regression models rather than this approach because we did not record the exact occurrence time of each outcome.

\section{Future studies}

Owing to the issue that manifestations of DM in patients with COVID-19 pose a considerable clinical challenge, further multicenter studies on different ethnicities are warranted. Furthermore, understanding the essential impacts of DM on the prognosis of COVID-19 needs more observations to be continued longitudinally and interpreted through time; thus, more similar studies can help to bring out the importance of this problem.

\section{Conclusion}

In summary, we found that in patients with DM, lower oxygen saturation, higher admission temperature, and higher serum urea, are independent predictors of in-hospital mortality for COVID-19. Patients with DM progressed to more severe disease with a significantly higher mortality rate than other patients. In addition, there is an association between uncontrolled DM status and severe disease manifestation and incurring ACI and ALI during admission. Overall, this study proved that DM might be a risk indicator for poor prognosis 
in COVID-19. Besides, close monitoring of admission oxygen saturation, temperature, and serum urea might have a proactive effect on understanding the progression of COVID-19 in patients with DM.

Supplementary Information The online version contains supplementary material available at https://doi.org/10.1007/s40200-020-00701-2.

Acknowledgments We acknowledge all health-care workers involved in the diagnosis and treatment of patients in Sina Hospital. We are indebted to the Research Development Center of Sina Hospital for its support. The authors are grateful to Mrs. Masoumeh Taleh, Mr. Saeed Hejrani, and members of the COVID-19 Crisis Committee of the Sina Hospital for their help and consult.

Authors' contributions MP: conceptualization, methodology, writing Original Draft, supervision, project administration. MK: formal analysis, investigation, data curation, writing - Original Draft. SK: conceptualization, methodology, investigation, visualization. MM: methodology, validation, supervision. MMA: validation, investigation, visualization. MS: investigation, data curation, visualization. SK: formal analysis, investigation, data curation, writing - Original Draft. MT: data curation, supervision. HA: conceptualization, validation, investigation, data curation, writing - Original Draft, Supervision, project administration. RSM: validation, investigation, supervision. FM: software, investigation, data curation, writing - Original Draft. NHA: software, investigation, data curation, visualization. SZ: investigation, visualization, supervision. MF: investigation, validation, supervision. ZA: investigation, data curation, visualization. $\mathbf{M E}^{*}$ : conceptualization, methodology, formal analysis, writing - Original Draft, supervision, project administration.

Funding This study has been supported by Tehran University of Medical Sciences (Grant number: 99-1-101-47211 to HA). The funding source had no role in the study design, data collection, data analysis, data interpretation, writing of the manuscript, or decision of submission.

Data availability Data are available upon a reasonable request to the corresponding author.

\section{Compliance with ethical standards}

Conflict of interest The authors declare that they have no conflict of interest.

Code availability Not applicable.

\section{References}

1. World Health Organization. WHO Coronavirus Disease (COVID19) Dashboard. 2020. https://covid19.who.int/. Accessed November, 8, 10:28am CET 2020.

2. Worldometer. COVID-19 CORONAVIRUS PANDEMIC. https:// www.worldometers.info/coronavirus/. Accessed November, 8, 13: 42 GMT 2020.

3. Chen N, Zhou M, Dong X, Qu J, Gong F, Han Y, et al. Epidemiological and clinical characteristics of 99 cases of 2019 novel coronavirus pneumonia in Wuhan, China: a descriptive study. Lancet. 2020;395(10223):507-13.

4. Yang J, Zheng Y, Gou X, Pu K, Chen Z, Guo Q, et al. Prevalence of comorbidities and its effects in patients infected with SARS-CoV-2: a systematic review and meta-analysis. International journal of infectious diseases : IJID : official publication of the International Society for Infectious Diseases. 2020;94:91-5. https://doi.org/10. 1016/j.ijid.2020.03.017.

5. Badawi A, Ryoo SG. Prevalence of comorbidities in the Middle East respiratory syndrome coronavirus (MERS-CoV): a systematic review and meta-analysis. International journal of infectious diseases : IJID : official publication of the International Society for Infectious Diseases. 2016;49:129-33. https://doi.org/10.1016/j.ijid. 2016.06.015.

6. Wu ZH, Tang Y, Cheng Q. Diabetes increases the mortality of patients with COVID-19: a meta-analysis. Acta Diabetol. 2020:16. https://doi.org/10.1007/s00592-020-01546-0.

7. Singh AK, Singh R. Does poor glucose control increase the severity and mortality in patients with diabetes and COVID-19? Diabetes \& metabolic syndrome. 2020;14(5):725-7. https://doi.org/10.1016/j. dsx.2020.05.037.

8. GBD Compare. Institute for Health Metrics and Evaluation. https:// vizhub.healthdata.org/gbd-compare/. Accessed November, 8, 2020.

9. Rossi G, Association AD. Diagnosis and classification of diabetes mellitus. Diabetes Care. 2018;33(Suppl 1):S62-S9.

10. Allard R, Leclerc P, Tremblay C, Tannenbaum T-N. Diabetes and the severity of pandemic influenza a (H1N1) infection. Diabetes Care. 2010;33(7):1491-3.

11. Iacobellis G. COVID-19 and diabetes: can DPP4 inhibition play a role? Diabetes Res Clin Pract. 2020;162:108125. https://doi.org/10. 1016/j.diabres.2020.108125.

12. Milionis C, Milioni SO. A brief analysis and hypotheses about the risk of COVID-19 for people with type 1 and type 2 diabetes mellitus. Journal of diabetes and metabolic disorders. 2020:1-5. https://doi.org/10.1007/s40200-020-00592-3.

13. Mantovani A, Byrne CD, Zheng MH, Targher G. Diabetes as a risk factor for greater COVID-19 severity and in-hospital death: a metaanalysis of observational studies. Nutrition, metabolism, and cardiovascular diseases : NMCD. 2020;30(8):1236-48. https://doi.org/ 10.1016/j.numecd.2020.05.014.

14. COVID-19 Epidemiology Committee. COVID-19 epidemiology. 2020. https://behdasht.gov.ir/. Accessed November, 8, 2020.

15. Clinical management of COVID-19. World Health Organization. https://www.who.int/publications/i/item/clinical-management-ofcovid-19. Accessed November 8, 2020.

16. Talebpour M, Hadadi A, Oraii A, Ashraf H. Rationale and Design of a Registry in a Referral and Educational Medical Center in Tehran, Iran: Sina Hospital Covid-19 Registry (SHCo-19R). Advanced Journal of Emergency Medicine. 2020;4(2s):e53-e.

17. 2. Classification and Diagnosis of Diabetes: Standards of Medical Care in Diabetes-2020. Diabetes Care. 2020;43(Suppl 1):S14-s31. doi:https://doi.org/10.2337/dc20-S002.

18. Carretero OA, Oparil S. Essential hypertension. Part I: definition and etiology. Circulation. 2000;101(3):329-35. https://doi.org/10. 1161/01.cir.101.3.329.

19. Ranieri VM, Rubenfeld GD, Thompson BT, Ferguson ND, Caldwell E, Fan E, et al. Acute respiratory distress syndrome: the Berlin definition. Jama. 2012;307(23):2526-33. https://doi.org/10. 1001/jama.2012.5669.

20. Adams J 3rd, Bodor GS, Davila-Roman VG, Delmez JA, Apple FS, Ladenson J, et al. Cardiac troponin I. A marker with high specificity for cardiac injury circulation. 1993;88(1):101-6.

21. Makris K, Spanou L. Acute kidney injury: definition, pathophysiology and clinical phenotypes. The Clinical biochemist Reviews. 2016;37(2):85-98.

22. Dufour DR, Lott JA, Nolte FS, Gretch DR, Koff RS, Seeff LB. Diagnosis and monitoring of hepatic injury. I. Performance characteristics of laboratory tests. Clin Chem. 2000;46(12):2027-49. 
23. Wu Z, McGoogan JM. Characteristics of and important lessons from the coronavirus disease 2019 (COVID-19) outbreak in China: summary of a report of 72314 cases from the Chinese Center for Disease Control and Prevention. Jama. 2020;323(13): 1239-42. https://doi.org/10.1001/jama.2020.2648.

24. Hussain A, Bhowmik B, do Vale Moreira NC. COVID-19 and diabetes: Knowledge in progress. Diabetes research and clinical practice. 2020;162:108142. https://doi.org/10.1016/j.diabres.2020. 108142.

25. Xu H, Zhong L, Deng J, Peng J, Dan H, Zeng X, et al. High expression of ACE2 receptor of 2019-nCoV on the epithelial cells of oral mucosa. International journal of oral science. 2020;12(1):8. https://doi.org/10.1038/s41368-020-0074-x.

26. Soleimani A, Kazemian S, Karbalai Saleh S, Aminorroaya A, Shajari Z, Hadadi A, et al. Effects of angiotensin receptor blockers (ARBs) on in-hospital outcomes of patients with hypertension and confirmed or clinically suspected COVID-19. Am J Hypertens. 2020. https://doi.org/10.1093/ajh/hpaa149.

27. Yang JK, Lin SS, Ji XJ, Guo LM. Binding of SARS coronavirus to its receptor damages islets and causes acute diabetes. Acta Diabetol. 2010;47(3):193-9. https://doi.org/10.1007/s00592-009-0109-4.

28. Hodgson K, Morris J, Bridson T, Govan B, Rush C, Ketheesan N. Immunological mechanisms contributing to the double burden of diabetes and intracellular bacterial infections. Immunology. 2015;144(2):171-85. https://doi.org/10.1111/imm.12394.

29. Chee YJ, Tan SK, Yeoh E. Dissecting the interaction between COVID-19 and diabetes mellitus. Journal of diabetes investigation. 2020;11(5):1104-14. https://doi.org/10.1111/jdi.13326.

30. Shi Q, Zhang X, Jiang F, Zhang X, Hu N, Bimu C, et al. Clinical characteristics and risk factors for mortality of COVID-19 patients with diabetes in Wuhan, China: a two-center. Retrospective Study Diabetes Care. 2020;43(7):1382-91. https://doi.org/10.2337/dc200598.

31. Deng Y, Liu W, Liu K, Fang YY, Shang J, Zhou L, et al. Clinical characteristics of fatal and recovered cases of coronavirus disease 2019 in Wuhan, China: a retrospective study. Chin Med J. 2020;133(11):1261-7. https://doi.org/10.1097/cm9. 0000000000000824.

32. Pagliaro P, Penna C. ACE/ACE2 ratio: a key also in 2019 coronavirus disease (Covid-19)? Frontiers in medicine. 2020;7:335. https://doi.org/10.3389/fmed.2020.00335.

33. Liu W, Tao ZW, Wang L, Yuan ML, Liu K, Zhou L, et al. Analysis of factors associated with disease outcomes in hospitalized patients with 2019 novel coronavirus disease. Chin Med J. 2020;133(9): 1032-8. https://doi.org/10.1097/cm9.0000000000000775.

34. Xie J, Covassin N, Fan Z, Singh P, Gao W, Li G, et al. Association between hypoxemia and mortality in patients with COVID-19. Mayo Clin Proc. 2020;95(6):1138-47. https://doi.org/10.1016/j. mayocp.2020.04.006.

35. Cheng Y, Luo R, Wang K, Zhang M, Wang Z, Dong L, et al. Kidney disease is associated with in-hospital death of patients with COVID-19. Kidney Int. 2020;97(5):829-38. https://doi.org/10. 1016/j.kint.2020.03.005.

36. Guo W, Li M, Dong Y, Zhou H, Zhang Z, Tian C et al. (2020) Diabetes is a risk factor for the progression and prognosis of COVID-19. Diabetes/metabolism research and reviews. e3319. doi:https://doi.org/10.1002/dmrr.3319.
37. Zhang Q, Wei Y, Chen M, Wan Q, Chen X. Clinical analysis of risk factors for severe COVID-19 patients with type 2 diabetes. J Diabetes Complicat. 2020;34(10):107666. https://doi.org/10.1016/ j.jdiacomp.2020.107666.

38. Cheng A, Hu L, Wang Y, Huang L, Zhao L, Zhang C, et al. Diagnostic performance of initial blood urea nitrogen combined with D-dimer levels for predicting in-hospital mortality in COVID-19 patients. Int J Antimicrob Agents. 2020;56(3):106110. https://doi.org/10.1016/j.ijantimicag.2020.106110.

39. Shao M, Li X, Liu F, Tian T, Luo J, Yang Y. Acute kidney injury is associated with severe infection and fatality in patients with COVID-19: a systematic review and meta-analysis of 40 studies and 24,527 patients. Pharmacol Res. 2020;161:105107. https:// doi.org/10.1016/j.phrs.2020.105107.

40. Raoufi M, Khalili S, Mansouri M, Mahdavi A, Khalili N. Wellcontrolled vs poorly-controlled diabetes in patients with COVID19: are there any differences in outcomes and imaging findings? Diabetes Res Clin Pract. 2020;166:108286. https://doi.org/10.1016/ j.diabres.2020.108286.

41. Iacobellis G, Penaherrera CA, Bermudez LE, Bernal ME. Admission hyperglycemia and radiological findings of SARSCoV2 in patients with and without diabetes. Diabetes Res Clin Pract. 2020;164:108185. https://doi.org/10.1016/j.diabres.2020. 108185.

42. Zhang Y, Li H, Zhang J, Cao Y, Zhao X, Yu N, et al. The clinical characteristics and outcomes of patients with diabetes and secondary hyperglycaemia with coronavirus disease 2019: a single-Centre, retrospective, observational study in Wuhan. Diabetes Obes Metab. 2020;22(8):1443-54. https://doi.org/10.1111/dom.14086.

43. Cuschieri S, Grech S. COVID-19 and diabetes: the why, the what and the how. J Diabetes Complicat. 2020;34(9):107637. https://doi. org/10.1016/j.jdiacomp.2020.107637.

44. Li X, Xu S, Yu M, Wang K, Tao Y, Zhou Y, et al. Risk factors for severity and mortality in adult COVID-19 inpatients in Wuhan. J Allergy Clin Immunol. 2020;146(1):110-8. https://doi.org/10. 1016/j.jaci.2020.04.006.

45. Zhu L, She ZG, Cheng X, Qin JJ, Zhang XJ, Cai J, et al. Association of Blood Glucose Control and Outcomes in Patients with COVID-19 and Pre-existing Type 2 Diabetes. Cell metabolism. 2020;31(6):1068-77.e3. https://doi.org/10.1016/j.cmet.2020. 04.021 .

46. Huang I, Lim MA, Pranata R. Diabetes mellitus is associated with increased mortality and severity of disease in COVID-19 pneumonia - a systematic review, meta-analysis, and meta-regression. Diabetes \& metabolic syndrome. 2020;14(4):395-403. https://doi. org/10.1016/j.dsx.2020.04.018.

47. Barrera FJ, Shekhar S, Wurth R, Moreno-Pena PJ, Ponce OJ, Hajdenberg M, et al. Prevalence of Diabetes and Hypertension and Their Associated Risks for Poor Outcomes in Covid-19 Patients. Journal of the Endocrine Society. 2020;4(9):bvaa102. https://doi.org/10.1210/jendso/bvaa102.

Publisher's note Springer Nature remains neutral with regard to jurisdictional claims in published maps and institutional affiliations. 